On a Modification of Mance's Method of measuring Battery Resistance

This content has been downloaded from IOPscience. Please scroll down to see the full text. 1875 Proc. Phys. Soc. London 2145

(http://iopscience.iop.org/1478-7814/2/1/314)

View the table of contents for this issue, or go to the journal homepage for more

Download details:

IP Address: 132.174.255.116

This content was downloaded on 01/10/2015 at 01:04

Please note that terms and conditions apply. 
XIV. On a Modification of Mance's Method of measuring Battery Resistance. By OLIver J. Lodge, B.Sc.

[Plate II.]

THE modification. here suggested consists simply in using a galvanometer and condenser instead of a galvanometer alone, so as to detect variations in difference of potential instend of variations in current.

By this change it is converted into a strictly null method. Moreover it is now possible entirely to get rid of the effects of variations in the electromotive force of the battery, which are very annoying in any of the ordinary methods and prevent accurate measure. This is accomplished by breaking the galvanometer-circuit the instant after the battery is short-circuited. Fig. 1 (Pl. II.) is a diagram of the connexions for measuring the resistance of the battery $d$, with the keys shown on a large scale: $m$ partially short-circuits the battery when depressed; $n$ closes the galvanometer and condenser circuit unless depressed. The two keys are electrically independent; but the stand of the upper one is balanced so as to rest partly on the spring of the lower one (which must be strong). On depressing the upper key, the first effect is to close the circuit marked $r$ at the point $m$; the second, and immediately succeeding, effect is to break the circuit marked $g$. at the point $n$. The same object would be accomplished more conveniently by a single double-contact key made on purpose, as shown in fig. 4 . The object of the double key is fully explained below. A B C D represents a box of resistance-coils; $a$ and $b$ are large and equal resistances; and $c$ will be equal to $d$, the resistance of the battery, whenever the galvanometer-needle is unaffected by pressing down the keys.

\section{Resistance-measurements in general.}

Consider the arrangement of six conductors joining four points (commonly known as the Wheatstone's bridge) as forming the edges of a tetrahedron or triangular pyramid (fig. 2). It is obvious, (1) that, as far as position is concerned, every conductor has precisely the same properties as any other, and (2) that any one conductor is adjacent to four of the others and 
opposite to the remaining one. Call the resistances of pairs of opposite ones $a$ and $c, b$ and $d, r$ and $g$, and let electromotive forces be caused to act in any manner through any of them ; then it can be shown that when $a c=b d, r$ and $g$ are "conjugate conductors," or that variations in the conductor $r$ have no effect whaterer on the current in $g$, and vice versî, no matter whether these variations are simple changes of resistance or the introduction of new electromotive force.

By ascertaining, then, whether the insertion or removal of hatteries at $r$ has any effect on a galvanometer at $g$, one can ohserve whether the relation $a c=b d$ is or is not fulfilled, and can change one of these resistances until it is. For the case when $a, b, c$, and $d$ are simple metallic conductors, this is Wheatstone's method of comparing their resistances.

Again, reciprocally, when this relation is fulfilled, no change in $g$ can affect the current through the battery in $r$; and therefore, if this battery in $r$ is the only electromotive force in action, a change in the resistance of $g$ does not affect the current at all anywhere. Hence a galvanometer in, say, $d$ will show a constant deflection whether the resistance of $g$ is 0 or $\infty$, whenever $a c=b d$; and this is Thomson's method of measuring the resistance of the galvanometer $d$.

Further, from what has been silid, there is no objection to an existence of electromotive force in any or all of the conductors, provided it remains constant; for it will be equally possible to observe whether changes in $r$ (of any sort) have any effect on the current in $g$; and if not, then $a c=b d$, as before. For the case when $d$ is a battery of constant electromotive force, this is Mance's method of determining its resistance.

But it must now be observed that although changes in $r$ may have no effect on the current in $g$, they must affect very essentially the current every where else, and therefore through the battery $d$. This battery ought, then, to preserve its electromotive force constant in spite of variations taking place in the strength of its current-a thing which no known battery is capable of doing. The electromotive force of every battery is really a function of the current it is producing and of the time it has lasted. In cells called constant the dependence of electromotive force on current and time is only slight; but in none dues it disappear. 
This fact that the current * and consequently, to some extent, the electromotive force of the battery are made to vary by the process of measuring its resistance, constitutes a great apparent defect of the method; but it is an irremediable defect, and is not peculiar to this particular method. It is in fact impossible to measure the resistance of a battery without rarying the strength of the current passing through it, by any method founded, as all our methods are, on a measurement of current or of difference of potential. In other words, just as it is impossible to measure any resistance whatever without the passage of a current through the resisting body, although it is quite easy to measure an electromotive force without any current circulating through the electromotor, so, although a current of constant strength is sufficient to give a measure of the resistance of a homogeneous conductor, such as a inetallic wire of uniform material, or a homogeneous liquid, or any thing else which contains no internal electromotive force, yet a variable strength of current is necessary to determine the resistance of an electromotor.

And the reason of this is apparent, viz. that the opposition experienced by a current in passing through an electromotor is of two kinds-one due to the proper olmic resistance, the other due to the electromotive force ; and with only one strength of current it is no more possible to tell how much of the opposition is resistance and how much is electromotive force, than it is to obtain the values of two unknown quantities from one equation. We may either take two measures of the strength of current and then eliminate one of the unknown quantities algebraically, or we may use a contrivance (like Mance's method) by which one of them (viz. electromotive force) is eliminated electrically; but two strengths of current are just as essential in the latter case as in the former, as also it is just as necessury that the two unknown quantities shall remain constant. It is possible that the resistance, as well as the electromotive force, of a battery does not accurately fulfil this condition, but that it raries to some extent with the current;

* Professor Clerk Maxwell, in describing this method ('Electricity and Magnetisn,' i. p. 411), says that " the current in the battery is not in any way interfered with during the operation :" but this must be a mistake. 
in so far as it does this, however, it is not a definite thing, and is incapable of accurate measurement.

I have entered into this matter at some length because the slip in Maxwell is getting repeated in other books ( $c f$. Cumming's admirable 'Introduction to the Theory of Electricity,' p. 162); and it is as well to get clear on the subject.

The difference of potential required to force a current of strength $\mathrm{C}$ through an electromotor of resistance $\mathrm{R}$ and internal electromotive force $\epsilon$ is

$$
\mathrm{E}=\mathrm{RC} \pm \epsilon
$$

Various methods may be applied to measure $\mathrm{E}$ and $\mathrm{C}$; but no observation of a single value of $E$ and $C$ can determine $R$ unless $\epsilon$ is known. Another observation with a different value of $\mathrm{E}$ and $\mathrm{C}$ must be made; and then $\epsilon$ can either be eliminated directly, or one can employ an indirect means of effecting its elinination, provided it remain constant. (If it does not remain constant, and if the law of its variation is unknown, no amount of experiments can eliminate it.) It is true that a single strength of current will suffice to determine $R$ after $\epsilon$ is known; but in the determination of $\epsilon$ another and quite different strength of current (viz. zero) was employed.

A curious illustration of the impossibility of measuring the resistance of an electromotor by means of a constant current was noticed the other day in the physical laboratory at University College by Mr. H. F. Morley, who has found that the current produced by a certain form of gas-battery is, within very wide limits, almost independent' of the resistance of its circuit. He endeavoured to measure the internal resistance of this battery by means of its own current, but found it quite impracticable.

Variation of the Electromotive Force of a Battery.

In what precise way the electromotive force of an ordinary cell depends on the current passing through it and on the time that current has lasted, I am not aware of any experiments which afford us information. But a law like the following seems not improbable.

$$
\mathrm{F}=\Lambda e^{-\frac{p t}{\bar{n}}}+\mathrm{B}, \quad . \quad \cdot \quad \cdot \quad . \quad .
$$


where $t$ is the time the cell has been in action through the resistance $R$; so that the rate of change of $E$ is proportional to the excess of the strength of current it is producing over the minimum strength to which it will ultimately fall, or

$$
\frac{d \mathrm{E}}{d t}=-p \frac{\mathrm{E}-\mathrm{B}}{\mathrm{R}}
$$

$p$ is a number which may be constant, or it may be a function of the current or of $t$; but for a cell making any pretensions to constancy, it must be small. $A$ and $B$ are constants such that $A+B$ is the initial and $B$, the final, value of $E$.

At any rate we may, I think, reasonably assume that $\mathrm{E}$ is not affected instantaneously, however much the resistance of the circuit $R$ is changed, but that it takes a certain time to change its value appreciably; consequently, if we only change $\mathbf{R}$ for an instant of time and then restore it to its original value, $\mathrm{E}$ may be regarded as constant. It is this fact, $\mathrm{I}$ apprehend, which gives Mance's method its practical value, and renders it superior to the somewhat similar methods of Siemens and Thomson, because in it the change of resistance of $r$ can be made very rapidly without disturbing the galvanometer, and need only last a few scconds. The shortest time, however, is sufficient for some variation to take place in the battery; and accordingly a kick of the needle is usually observed, like that produced by an extra current, which is very annoying. The modification which I have to propose, however, renders possible so great a virtual diminution of the period of contact that this disappears.

\section{Modification of Mance's Method.}

There is also a practical objection to the ordinary form of Mance's method, not relating to its essentials, but to its sensibility and convenience, which the modification is intended entirely to remove. It is this :-The galvanometer in $g$, whose function it is to indicate any change in the current in that branch, has always a certain current passing through it, and its needle is therefore deflected nore or less, according to the sensibility of the galvanometer ; but the current produced by an ordinary cell whose resistance one wishes to measure is usually such as one does not care to pass through a delicate 
instrument, even if the excessive deviation it produces be corrected by external magnets. A rough galvanometer is therefore generally employed, and the needle is brought back reasonably near its mean position by magnets placed near it. But the needle being thus constrained by immersion in a powerful magnetic field, is by no means under favourable conditions, and only comparatively large changes in the current can be indicated by it. To remedy this defect and to make the method a null one, my first idea was to use a differential galvanometer and to send through its second wire a current from an auxiliary battery equal and opposite to the current produced in its first wire by the cell whose resistance is being determined, so as really to neutralize instead of merely to overpower its action on the needle. Or, without using a differential galvanometer, we may balance the electromotive force in the galvanometer circuit by means of an auxiliary closed battery circuit after the manner of Poggendorff. If either of these arrangements be adopted, we can use a sensitive 'Thomson's galvanometer, and its needle may be as nearly astatic as we choose. But it is not easy to get the two batteries under such similar conditions that they shall constantly oppose one another exactly ; and though these arrangements may be useful in some cases, they are rather complicated and the adjustments somewhat difficult to make.

The next alteration which suggested itself consisted in interpasing a condenser in the galvanometer circuit (see fig. 3). This effectually prevents any continuous circulation of electricity in that branch; and the galvanometer therefore remains at zero after the condenser has acquired its full charge; but any rariation in this charge is indicated by a throw of the galvanometer-needle proportional to the amount of variation. The quantity of electricity flowing into or out of the condenser through the galvanometer-coil will be equal to the variation of potential, $\mathrm{Y}$, taking place between its terminals multiplicd by $S$, its statical capacity ; and the throw of the galvanometerneedle $\alpha$ will be proportional to this quantity multiplied by the galvanometer-constant $\Gamma$, which depends directly on the nunber of turns of wire on it. The resistance of the galvanometer is quite immaterial. If $\mathrm{H}$ is the strength of the magnetic field in which the needle hangs and $\mathrm{T}$ the time of a complete 
oscillation of the needle in that field, we have

$$
\sin \frac{\alpha}{2}=\frac{\pi \Gamma S Y}{\mathrm{HT}^{\top}} \cdot \text {. . . . . . . }
$$

By using, therefore, a galvanometer with a very large number of turns, and a condenser of great capacity, one can increase the sensitiveness of the method to any extent.

The investigation of the distribution of currents throughout the circuit becomes very simple now that there is no continuous current through the branch $g$. The connexions are shown in fig. 3 , where $\mathrm{AC}$ is the branch $r$, whose resistance can be changed at pleasure from infinity to something near zero. Let A, B, C, D, be the potentials of the four corners; let $d$ be the resistance of the battery we wish to measure, $\epsilon$ its electromotive force, and $u$ the strength of the current passing through it. We want the difference of potential $B-D$ to be wholly independent of the potentials of $A$ and $C$, which will be altered by changing $r$. Now as there is no current through $g$, we have the same current passing through $b$ as through $a$-that is,

$$
\frac{\mathrm{A}-\mathrm{B}}{a}=\frac{\mathrm{B}-\mathrm{C}}{b} \text {, or } \mathrm{B}=\frac{\mathrm{A} b+\mathrm{C} a}{a+b} ;
$$

similarly

$$
\mathrm{D}=\frac{(\mathrm{A}-\epsilon) c+\mathrm{C} d}{c+d}
$$

hence

$$
\mathrm{B}-\mathrm{D}=\frac{(\mathrm{A}-\mathrm{C})(b d-a c)+\epsilon c(a+b)}{(a+b)(c+d)}, .
$$

which shows that the difference of potentials between the terminals of the condenser is independent of the potentials $A$ and $\mathrm{C}$ as soon as the condition $b d-a c=0$ is satisfied.

We may conveniently write the above expression in terms of the strength of the current $u$ passing through the battery $d$; thus, since $\mathrm{A}-\mathrm{C}=\epsilon-(c+d) u$,

$$
\mathrm{B}-\mathrm{D}=\frac{(a c-b d) u+\epsilon b}{a+b}
$$

So, if $a c=l d$, the difference of potential $\mathbf{B}-\mathbf{D}$ is quite independent of the current through the cell (except in so far as the 
electromotive force $\epsilon$ depends upon it) and is equal to $\frac{\epsilon b}{a+b}$ or $\frac{\epsilon c}{c+d}$, which are the same thing.

The current $u$ is of course dependent on the resistance $r$ of the branch $\mathrm{AC}$, being

$$
u=\frac{(a+b+r) \epsilon}{(a+b)(c+d)+r(a+b+c+d)} ; . .
$$

so we may also write the above difference of potential in terms of this resistance $r$, thus :-

$$
\mathrm{B}-\mathrm{D}=\epsilon \cdot \frac{(a+b) c+r(b+c)}{(a+b)(c+d)+r(a+b+c+d)} .
$$

All the differential coefficients of this with respect to $r$ contain the factor $a c-b d$; consequently when this factor vanishes this quantity is independent of $r$.

\section{Conditions for Sensitiveness.}

To find out what are the values of $a, b$, and $c$ which give the greatest sensitiveness, we can subtract the value of $B-D$ when $r$ is infinite from its value when $r$ is zero, and can make this quantity a maximum when the condition $a c=b d$ is nearly fulfilled. The quantity which has to be a maximum is

$$
y=(\mathrm{B}-\mathrm{D})_{0}-(\mathrm{B}-\mathrm{D})_{\infty}=\frac{\epsilon(a c-b d)}{(c+d)(a+b+c+d)} .
$$

The resistance $d$ is supposed to be given; so let us define the others with reference to it, putting

$$
c=\lambda d, a=\mu d, \text { and } l \bumpeq \lambda \mu d=\lambda \mu(1-z) d,
$$

where $z$ is a small quantity; then the above quantity becomes

$$
y=\frac{\lambda \mu z \epsilon}{(\lambda+1)^{2}(\mu+1)}
$$

Considered as a function of $\lambda$, this is a maximum when $\lambda=1$; it has in fact the same value whether $\lambda=n$ or $\frac{1}{n}$. Considered as a function of $\mu$, it has no maximum, but it is greatest when $\mu$ is infinite, though it does not incrense fast after $\mu$ is tolerably large; the curve is, in fact, a rectangular hyperbola with asymptotes $y=1$ and $\mu=-1$; and 1 is its greatest value for prositive values of $\mu$. Accordingly the most sensitive arrange- 
ment is obtained when $\lambda=1$ and when $\mu=\infty$-that is to say, when $c$ is equal to $d$ (the resistance to be measured), and when $a$ and $b$ are equal and as large as convenient. When these arrangements are made, the maximum value of $y$, or the change in the difference of potential between the terminals of the condenser brought about by depressing the key, is, when $c$ is nearly equal to $d$,

$$
\mathrm{Y}=\frac{1}{4} \epsilon z \quad \text { or }=\frac{\epsilon}{4} \cdot \frac{c-d}{d}
$$

and this is the quantity to be inserted in equation (3).

The sensitiveness is evidently directly proportional to the electromotive force of the cell : but it is independent of its resistance; $i . e$. a high resistance is measured with as great proportional accuracy as a low one. But it must be remembered that when the resistance to be measured is great, the resistances $a$ and $b$ should be as great also; if they are not as great as $d$, the sensitiveness falls off very appreciably. But, as said before, there is really no limit to the sensitjveness of the method; for the size of the condenser and the length of wire on the galvanometer may be increased to any extent.

\section{Practical Details.}

The condenser I have used is a small standard one with the dielectric of mica; and it has a capacity of slightly over one micro-farad. The galvanometer is a Thomson astatic by Elliott, with a resistance of about $7000 \mathrm{ohms}$. The two branches $a$ and $b$ of the resistance-coils, forming the equal arms of the bridge, were $1000 \mathrm{ohms} \mathrm{each,} \mathrm{being} \mathrm{the} \mathrm{largest} \mathrm{resistance}$ conveniently available in the box of resistance-coils used. But when the resistance to be measured is large (say over 500 ohms) it is better to have $a$ and $b$ greater than this; and they may then be made of Muirhead's carbon-paper (fig. 4). A strip 2 feet long by half an inch broad will have a resistance of about 14000 ohms; and the galvanometer terminal B may be connected with its middle so as to divide it into two halves representing $a$ and $b$. Exact equality in the two arms is not essential, as it is easy (and, indeed, generally advisable) to eliminate any errors of this sort by a method analogous to double weighing. Connect $a$ and $b$ to a commutator in such a way that it is easy to interchange them end for end (see fig. 4), and balance the 
resistance $d$ by the coils $c$; then interchange $a$ and $b$ and balance again; this time we may require an amount $c^{\prime}$. Then it is easy to see that $d=\sqrt{ }\left(c c^{\prime}\right)$, whatever the ratio of $a$ to $b$; for in the first case we have $d: c=a: b$, and in the second $d: c^{\prime}=b: a$. If $c$ and $c^{\prime}$ are nearly equal, their arithmetic mean may be taken instead of their geometric, as being easier to calculate.

\section{Use of a Double-wire Galvanometer as an Electrometer.}

When a differential galvanometer, or a galvanometer with two long fine wires wound side by side, is employed, a separate condenser is not absolutely necessary ; for the galvanometer itself has a certain capacity, and in order to charge one of its wires up to the potential $B$, and the other down to the potential $\mathrm{D}$, a certain quantity of electricity must flow into the one wire and out of the other, and any variation in this quantity will affect the needle (though the galvanometer-constant has only half its ordinary value). Even when a separate condenser is used the capacity of the differential galvanometer may be taken advantage of, by connecting the terminals of the condenser to its two middle screws (instead of joining them directly to each other by a wire and inserting the condenser as in fig. 3), so that both condenser and galvanometer get charged instead of only the condenser. The defect of this method is, that the insulation between two silk-covered wires is not very perfect, and there is a slight leakage, which maintains a slight continuous deflection of the needle when the two outer screws are joined up to a battery; moreover the statical capacity of an ordinary fine-wire differential galvanometer is not very great.

But I think it may be often convenient to use a double-wire galvanometer as an electromoter in this way. For instance, rapidly to compare the electromotive force of any number of cells, join them up to the outer screws of the galvanometer with disconnected wires one after the other ; the kick in each case measures the electromotive force of the cell. It might also be used to measure very high resistances. It is quite possible, and indeed very probable, that this method has been suggested before. 
Elimination of Irariutions in Electromotive Force.

It has been stated above that if only momentary variations are made in the resistance $r$, or in the value of $u$, we can consider $\epsilon$, the electromotive force of the battery, constant. The plan I adopt is to make the effective variation of $r$, or the variation which is to have any influence on the galvanometer, very short indeed. And this is done by arranging that the key $m$ which closes the circuit of $r$ shall break the galvanometer circuit $g$, the instant after, at the point $n$, as shown in figs. 1 and 4. For an instant, then, $u$ is varied; and if the resistances are not balanced so that $a c=b l$, a certain quantity of electricity will enter or leave the condenser through the galvanometer; but variations in $\epsilon$ (due to the changed $u$ ), which would produce the same effect on the galvanometer, no matter how much the resistances were balanced, have no time to take place before the galvanometer circuit is broken; and then no further change has any effect. This works very well in practice; and the resistance of a cell can be really determined when producing a current through a definite resistance, viz. $a+b+c+d$. This cannot be done accurately by any other method that I know of.

\section{Measurement of any Liquid Resistances.}

The method may be applied to determine the resistance of electrolytes in general. A long tube containing the electrolyte surrounded by a jacket of water at a known temperature is interposed in the battery circuit $d$, the battery being one whose resistance is small and can be depended on; and the resistance of the two together, battery and tube, is measured.

The tube is then removed, and the resistance of the battery determined alone; the difference of course gives the resistance of the electrolyte in the tube. The tube can then be filled with mercury and the measurement repeated. The amount of polarization of the electrodes is of no more consequence than the electromotive force of the battery; provided the gas given off is not allowed mechanically to obstruct the current; and the effect of variations in its amount are reduced to a minimum by the method just described for the battery. It is well to make the tube end in a couple of globular receivers with 
two necks, very much like Dewar's electrometer, and to plunge large electrodes into these globes (see T, fig. 4). Their position in the globes is not of very much consequence; neither is a bubble or two of gas on their surface; the principal part of the resistance is offered by the liquid in the tube. If a thermometer is kept in each receiver and the liquid be allowed to flow backwards and forwards through the tube once or twice before observing, its temperature may be known without sur. rounding it with a jacket. But of course this does not apply accurately when it is required to raise its temperature much above that of the aimosphere. Since the sensitiveness depends on the electromotive force of the battery, it is well to use one or two Grove's cells. Fig. 4 is a complete diagram of the connexions, showing the arrangement for interchanging $a$ and $b$ by means of a commutator, and also showing a key which will break the galvanometer circuit at the instant required and act instead of the extemporized arrangement of two keys depicted in fig. 1. The lower spring carries a block which presses up against a screw connected with the galvanometer, except when it is forced down by the upper spring. This block is insulated from the spring, which carries it, but is connected with the point $B$. The interral elapsing between the breaking contact at $m$ and the making at $n$ is, and must be, utterly inappreciable. For the shortest practicable interval is sufficient to allow the currents to adjust themselves, unless $a$ and $b$ are extravagantly large resistances; and if it is not exceedingly short, disturbances will occur due to variations in the battery.

The diagrams purposely show the galvanometer, and not the condenser, connected with the breaking-key $n$, because leakage of the condenser is sure to occur to some extent when its circuit is broken, and, in order that this may not in the least signify, one terminal of the galvanometer must be insulated.

University College, London. 